\section{SWNTs Used to Translocate RNA Polymer}

A critical component in the development of gene therapy involves transfection-the uptake of foreign DNA into cells. However, the physicochemical properties of DNA are themselves impediments to the transfer of DNA across cell membranes (translocation). Viral transfection vectors are currently most effective because viruses have a natural ability to introduce genetic material into cells. However, the immune responses to viruses frequently prevent successful gene delivery. Nonviral transfection vectors avoid this difficulty but suffer from low efficiency rates for nuclear membrane penetration and gene expression. Those few nonviral transfection vectors that have achieved high efficiency also exhibit cytotoxicity. Recently, P.C. Ke and colleagues from Clemson University have considered as transfection vectors single-walled carbon nanotubes (SWNTs) because of their large surface area, stability, flexibility, and biocompatibility.

As reported in the December 8, 2004, issue of Nano Letters (p. 2473; DOI: $10.1021 /$ nl048326j), the researchers demonstrated the delivery of RNA polymer poly(rU) into breast cancer cells using SWNTs as transporters. The researchers synthesized SWNT bundles using the arcdeposition method. The prevalent SWNT diameter was about $1.4 \mathrm{~nm}$. SWNT-RNA poly $(\mathrm{rU})$ hybrids were formed through nonspecific binding, which allows the hybrid to dissociate into its two components upon delivery. The researchers fluorescently labeled SWNT-poly(rU) hybrids with propidium iodide (PI) and then incubated the hybrids with MCF7 breast cancer cells (cell membranes are known to be impermeable to PI). The researchers used as controls PI and PI-labeled poly(rU).

For imaging, the researchers used confocal fluorescence microscopy because it allows for axial discrimination of the labeled SWNT-poly(rU) hybrids on cell membranes, within cytoplasm or within the nucleus. The research team also employed radioisotopic labeling, cell enumeration, and a standard metabolic activity assay (MTS) to quantitatively evaluate direct cellular uptake and SWNT cytotoxicity. The researchers found the labeled SWNT-poly(rU) hybrids across the cellular and nuclear membranes of the MCF7 cells, while the controls were excluded. Furthermore, the research team showed, by means of cell growth and MTS assays, that SWNTs have no cytotoxicity for concentrations of up to $1 \mathrm{mg} / \mathrm{mL}$. Ke and co-workers said that their studies show the potential of
SWNTs as transporters for gene delivery. They believe that SWNTs and biomedicine can be more fully integrated once the mechanisms of SWNT translocation are deciphered and gene transfection assays with SWNT transporters are performed.

STEVEN TROHALAKI

\section{Fatigue Properties of Nanometer- Scale Copper Films Elucidated}

The current trend toward the development of small-scale structures in various areas of nanotechnology necessitates the understanding of materials properties at very small length scales. It is critical to determine mechanical properties and the underlying microstructural mechanisms in order to construct reliable structures for micro- and nanosystems. Several recent studies have demonstrated that fatigue properties of thin metal films, with thicknesses of less than a few micrometers, are significantly different from those of the bulk material. G.P. Zhang, of Max-PlanckInstitut für Metallforschung, Stuttgart, and the Institute of Metal Research, Chinese Academy of Sciences, Shenyang; C.A. Volkert, of Institut für Materialforschung II, Forschungszentrum Karlsruhe; and their co-workers have investigated in detail the fatigue properties and behavior of thin $(200 \mathrm{~nm}) \mathrm{Cu}$ films and compared the results with properties of much thicker $\mathrm{Cu}$ films. They report their findings in the January issue of the Journal of Materials Research (DOI: 10.1557/JMR.2005.0019).

The researchers deposited 200-nm-thick $\mathrm{Cu}$ films using magnetron sputtering onto dogbone-shaped polyimide substrates. The average grain size after annealing was determined to be $1.21 \mu \mathrm{m}$, with a standard deviation of $0.55 \mu \mathrm{m}$. Fatigue testing (cyclic loading and unloading) was performed with a constant total strain range of $1 \%$, an estimated plastic strain range of $0.8 \%$, and at $0.1 \mathrm{~Hz}$ frequency. For comparison, fatigue testing of 3.0- $\mu$ m-thick $\mathrm{Cu}$ films (1.45 $\mu \mathrm{m}$ average grain size) was conducted using a $1 \%$ total strain range and a $0.91 \%$ plastic strain range. The specimens were examined and characterized using focused ion-beam microscopy (FIB), scanning electron microscopy (SEM), and transmission electron microscopy (TEM).

The 200-nm-thick films showed only a few small extrusions on the surface and extensive cracking along grain boundaries and twin boundaries. The thicker $3.0 \mu \mathrm{m}$ films yielded more conventional larger extrusions/intrusions, with numerous cracks along the extrusions/intrusions and at the intersection of extrusions with grain boundaries. TEM revealed typical dislocation walls and cells within grains and near extrusions and twins for the $3.0 \mu \mathrm{m}$ films. In contrast, the $200 \mathrm{~nm}$ films showed individual dislocations rather than wall and cell structures. These results indicate that there is a transition in fatigue behavior with decreasing film thickness. As the thickness is reduced from the micron scale toward the submicron scale, dislocation cell structures disappear, extrusions become fewer and narrower, and cracking occurs along grain boundaries and twin boundaries. In other words, localized accumulation of plastic strain within grains is restricted, and the boundaries become the preferred site for damage (see Figure 1).

The researchers have suggested detailed mechanisms and models for these results based on the fact that dislocation nucleation and mobility are inhibited in small crystal volumes, which they suggest could account for the increased fatigue life observed with smaller sample dimensions. The results of this study provide the underlying cause for this increase. The researchers speculate that if the length scale is further reduced, inhibition of full dislocation plasticity could cause fatigue damage to be completely controlled by grainboundary-accommodated mechanisms.

GOPAL RAO
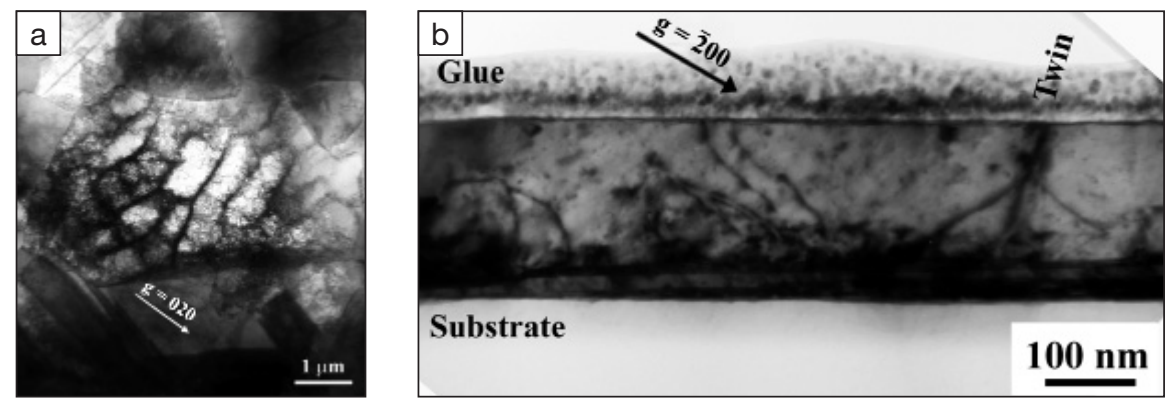

Figure 1. Transmission electron microscopy images show (a) the dislocation wall and cell structures within a grain for the $3.0 \mu \mathrm{m}$ Cu film and (b) a highly constrained dislocation motion and the absence of the dislocation structure for the 200-nm-thick Cu film. Reproduced with permission from the Journal of Materials Research 20 (1) (January 2005), DOI: 10.1557/JMR.2005.0019. (C) 2005 Materials Research Society. 